Journal of Advanced Dielectrics

Vol. 1, No. 4 (2011) 407-416

(C) World Scientific Publishing Company

DOI: $10.1142 /$ S2010135X11000549

\title{
CORRELATION BETWEEN THE ELASTIC AND THE VIBRONIC BEHAVIOR OF NANOSTRUCTURED TITANIA AND THEIR PRESSURE, SIZE, AND TEMPERATURE DEPENDENCE
}

\author{
X. J. LIU*, , L. K. PAN*, Z. SUN*, Y. M. CHEN ${ }^{\dagger}$, X. X. YANG ${ }^{\dagger}$, \\ L. W. YANG ${ }^{\dagger}$, Z. F. ZHOU ${ }^{\dagger}$ and CHANG Q. SUN ${ }^{\dagger}, \$$ \\ *Engineering Research Center for Nanophotonics $\&$ Advanced Instrument \\ Ministry of Education, Department of Physics \\ East China Normal University \\ Shanghai 200062, P. R. China \\ ${ }^{\dagger}$ Institute for Quantum Engineering and Micro-Nano Energy Technology \\ Key Laboratory of Low-Dimensional Materials and Application \\ Technology and Faculty of Materials and Optoelectronic Physics \\ Xiangtan University, Hunan 411105, P. R. China \\ $\$$ School of Electrical and Electronic Engineering \\ Nanyang Technological University \\ Singapore 639798, Singapore \\ §ecqsun@ntu.edu.sg
}

Received 29 April 2011

Revised 26 June 2011

\begin{abstract}
Correlation between the elastic and the vibronic behavior of $\mathrm{TiO}_{2}$ and their responses to the variation of crystal size, applied pressure, and measuring temperature has been investigated based on the bond order-length-strength correlation mechanism. Theoretical reproduction of the measurements clarified that: (i) the elastic modulus $(B)$ and the Raman shifts $(\Delta \omega)$ are strongly correlated and we can know either one of the $B$ or the $\Delta \omega$ from the other; (ii) the undercoordination induced cohesive energy loss and the energy density gain in the surface up to skin depth determines the size effect; (iii) bond expansion and bond weakening due to thermal vibration originates the thermally softened elastic modulus and the Raman shifts; and (iv) bond compression and bond strengthening results in the mechanically stiffened elastic modulus and the Raman shifts. With the developed premise, one can predict the changing trends of the concerned properties with derivatives of quantitative information of the atomic cohesive energy, binding energy density, Debye temperature, and nonlinear compressibility of the specimen.
\end{abstract}

Keywords: $\mathrm{TiO}_{2}$ nanostructures; elasticity; Raman shift; pressure; temperature.

${ }^{\S}$ Corresponding author. 


\section{Introduction}

Nanostructured Titania $\left(\mathrm{n}-\mathrm{TiO}_{2}\right)$ has attracted tremendous interest owing to their intriguing chemical and physical properties that the bulk counterpart does not demonstrate, ${ }^{1,2}$ and its many potential applications in photocatalysis, ${ }^{3}$ dyesensitized solar cells, ${ }^{4,5}$ sensor devices,${ }^{6}$ diluted magnetic semiconductors,${ }^{7}$ superhydrophobicity ${ }^{8}$ superhydrophilicity medium, ${ }^{9}$ etc. However, deeper insights into the physical origin and the correlation among various properties remain high challenge. The elastic and vibration properties of $\mathrm{TiO}_{2}$ under the applied stimuli of temperature $(T)$ and pressure $(P)$ and the variation of solid size $(K)$ are of instrumental importance from both fundamental and application points of view.

The bulk modulus $B$ (or the Young's modulus $Y \sim 3 B$ ) that is proportional to the binding energy density determines the performance of a material such as the extensibility, acoustic transmission velocity, Debye temperature, specific heat capacity, and thermal conductivity of the specimen. With the size reduction, the $B$ of $\mathrm{TiO}_{2}$ remains no longer the bulk constant but becomes tunable with the shape and feature size of the specimen. Under the applied $T$ and $P$, the $B$ also changes despite the artifacts arisen during the course of measurements such as the accuracy of size and surface passivation. Intensive investigations have revealed unexpectedly that the $Y$ of $\mathrm{TiO}_{2}$ ascends when the solid size is reduced from the infinitely large bulk to the atomic scale or it is placed under high pressure. ${ }^{2}$ However, the $B$ is softened at high temperature. ${ }^{10,11}$

The anatase $\mathrm{TiO}_{2}$ has a total of six Raman active modes of $3 \mathrm{E}_{\mathrm{g}}$ (at 144, 196, and $639 \mathrm{~cm}^{-1}$ wavelengths), $2 \mathrm{~B}_{1 \mathrm{~g}}\left(397\right.$ and $\left.519 \mathrm{~cm}^{-1}\right)$, and $1 \mathrm{~A}_{1 \mathrm{~g}}$ $\left(513 \mathrm{~cm}^{-1}\right)$ and rutile $\mathrm{TiO}_{2}$ has only four Raman active modes of $\mathrm{A}_{1 \mathrm{~g}}\left(612 \mathrm{~cm}^{-1}\right), \mathrm{B}_{1 \mathrm{~g}}\left(143 \mathrm{~cm}^{-1}\right), \mathrm{E}_{\mathrm{g}}$ $\left(447 \mathrm{~cm}^{-1}\right)$, and $\mathrm{B}_{2 \mathrm{~g}}\left(826 \mathrm{~cm}^{-1}\right)$. Generally, the frequency of the transverse optical (TO) phonon undergoes a red shift upon the radius $R$ of nanosolid being decreased and almost all the modes are stiffened under high pressure and softened at elevated temperatures. ${ }^{12-14}$ However, for the $\mathrm{TiO}_{2}$, the $\mathrm{A}_{1 \mathrm{~g}}$ $\left(612 \mathrm{~cm}^{-1}\right)$ mode of rutile phase undergoes a red shift while the $\mathrm{E}_{\mathrm{g}}\left(144 \mathrm{~cm}^{-1}\right)$ mode of anatase phase undergoes a blue shift when the solid size is reduced. ${ }^{14-17}$

Numerous models have been developed from various perspectives to illustrate the unusual elastic and vibration behavior in general under the conditions of size reduction, temperature, and pressure elevation. For the size-induced $Y$ elevation, mechanisms of surface tension, ${ }^{18}$ surface stiffness, ${ }^{19}$ surface relaxation, ${ }^{18}$ surface reconstruction, ${ }^{20}$ surface stress, ${ }^{21}$ bulk nonlinear elasticity, ${ }^{19}$ surface bond contraction, ${ }^{22}$ competition between electron redistribution and the lower-coordination on surfaces, ${ }^{23}$ and vacancy formation determined by the cohesive energy, ${ }^{24}$ surface energy density gain ${ }^{25}$ have been developed; for the size-induced $\Delta \omega$ softening, mechanisms of surface disorder, ${ }^{26}$ surface stress, ${ }^{27}$ quantum confinement, ${ }^{28}$ local heating effects ${ }^{29}$ or surface chemical passivation have been proposed; for the thermal effect, models have also been developed $^{30-32}$ with the involvement of the thermal expansion coefficient and the mode AndersonGrüneisen parameter. These models interpret the $T$ dependence of $\omega$ in terms of the anharmonic phonon decay, ${ }^{33}$ thermal expansion, ${ }^{34}$ phonon-phonon interaction, ${ }^{35}$ modifies phonon decay, ${ }^{36}$ and frequency perturbation mechanism. ${ }^{37,38}$ Generally, the size, $T$, and $P$ dependences of the $\omega$ are modeled as, ${ }^{37-41}$

$$
\left\{\begin{array}{l}
\Delta \omega(R)=\omega(R)-\omega(\infty)=A_{1}(a / R)^{\kappa} \\
\omega(T)=\omega(0)+\Delta \omega_{e}(T)+\Delta \omega_{d}(T) \\
\omega(P)=\omega(0)+A_{2} P+A_{3} P^{2}
\end{array}\right.
$$

where $\omega(0)$ is the Raman frequency measured at $0 \mathrm{~K}$, $\Delta \omega_{e}(T)$ is the contribution from the thermal expansion; $\Delta \omega_{d}(T)$ is due to the anharmonic coupling of phonons of other branches. $A_{2}$ and $A_{3}$ are the adjustable parameters without physical indication. $A_{1}$ and $\kappa$ are adjustable parameters used to fit the measured data. The $a$ is the lattice parameter that contracts with the solid dimension. ${ }^{42}$ For the blue shift, $A_{1}>0$ and $\kappa=1$, and $\omega(\infty)=0$.

Although the existing models could reproduce the measurements numerically, physical indications of the freely adjustable parameters need to be clear. A model that is able to correlate the $B$ and $\Delta \omega$ unifies their size, $T$, and $P$ effects is therefore greatly desired. Based on the bond order-length-strength (BOLS) correlation mechanism ${ }^{43}$ and the local bond averaging (LBA) approach, ${ }^{44}$ this presentation aims to show that such a model is feasible. In addition to the derived information of the atomic cohesive energy, binding energy density, Debye temperature, compressibility and the bulk modulus and their first-order derivatives, and the analytical correlation between the $B$ and $\Delta \omega$, the developed 
approach can improve our understanding of the unusual elastic and vibration behavior of $\mathrm{TiO}_{2}$. Theoretical reconciliation of the observations confirmed that: (i) the size effect arises from the undercoordination induced cohesive energy loss and the energy density gain in the surface up to skin depth; (ii) the thermally softened $B$ and $\Delta \omega$ results from bond expansion and bond weakening due to vibration; and, (iii) the mechanically stiffened $B$ and $\Delta \omega$ results from bond compression and bond strengthening due to mechanical work hardening.

\section{Principle}

\subsection{BOLS correlation and $L B A$ approach}

The BOLS correlation theory ${ }^{43}$ indicates that the broken bonds cause local bond contraction, bond strength gain, densification, and quantum entrapment of charge and energy. They hence modulate the local atomic cohesive energy, the binding energy density, and the Hamiltonian of the entire specimen and their relevant properties.

Numerically, the BOLS correlation is expressed as ${ }^{43}$

$$
\left\{\begin{array}{r}
d_{i} / d_{0}=c_{i}=2 /\left\{1+\exp \left[\left(12-z_{i}\right) / 8 z_{i}\right]\right\} \\
\quad \text { (bond contraction) } \\
E_{i} / E_{0}=c_{i}^{-m} \quad \text { (bond strengthening). }
\end{array}\right.
$$

The subscripts $i$ and 0 denote an atom in the $i$ th atomic layer and in the bulk as standard, respectively. The bond contraction coefficient varies only with the effective coordination number $\left(\mathrm{CN}\right.$, or $\left.z_{i}\right)$ of the atom of concern regardless of the nature of the bond. The index $m$ is the bond nature indicator that is not freely adjustable for a given material.

The theory has enabled the unification of atomic vacancies, adatoms, defects, atomic chains, atomic sheets, nanotubes, surface skins, nanocavities, and nanostructures of various shapes in the unusual mechanical, thermal, acoustic, chemical, electronic, dielectric, ferroelectric, optic, and magnetic properties and the transport dynamics of electrons and phonons. It has been clear that the tunable fraction of the undercoordinated atoms and the interaction between them dominate the size tunable properties at the nanoscale.

The LBA method ${ }^{44}$ connects the detectable properties of a substance directly to the identities (nature, order, length, strength) of a representative of all the bonds of the specimen and the response of these identities to the applied stimulus such as the coordination environment, pressure, temperature, etc.

Considering the contribution from the outermost three atomic layers, a core-shell configuration is implemented. Generally, both theory and experimental results show that the size trend of a quantity $Q(K)$ follows the linear dependence on the inverse of size $1 / K$ with $K$ being the dimensionless form of size, or the number of atoms lined along the radius of a sphere, ${ }^{44}$

$$
\begin{aligned}
& \frac{\Delta Q(K)}{Q(\infty)} \\
& \quad=\frac{1}{K} \begin{cases}\Delta_{q}=\sum_{i \leq 3} \tau c_{i}\left(\frac{q_{i}-q_{0}}{q_{0}}\right) & \text { (BOLS theory) } \\
B_{q} & \text { (Measurement) },\end{cases}
\end{aligned}
$$

where $\tau c_{i} / K$ is the surface-to-volume ratio representing the geometrical contribution from size $K$, dimensionality $\tau$, and the effective $\mathrm{CN}, c_{i}\left(z_{i}\right)$, which determines the size trend. $\Delta_{q}$ is a constant in each case; $q_{i}$ and $q$ are the density of $Q$ at the $i$ th atomic site and in the bulk. The term $\left(q_{i}-q_{0}\right) / q_{0}$ originate the size dependency. A particle at infinitely large, $1 / K \sim 0$ or the surface is neglected $q_{i}=q_{0}$, no size dependence would be observed. Therefore, ${ }^{25}$ any size trends are dictated by atoms in the surface skin up to three atomic layers in depth while atoms in the core interior make no contribution. $\tau=1,2$, and 3 corresponds to a thin plate, cylindrical rod, and spherical dot, respectively. The $i$ is counted from the outermost atomic layer inwards up to three. ${ }^{45}$ In the experimental $Q(K)-1 / K$ linear dependence, $B_{q}$ is the slope and the intercept in the $Y$ axis corresponds to the bulk value $Q(\infty)$. Equaling the BOLS derivatives with the measured size trend, we have the $B_{q}=\Delta_{q}$, which allows the unknown parameter such as $m$ to be determined.

This approach has enabled findings of: (i) the factors responsible for the thermo-mechanical behavior of atomic chains, nanotubes, nanowires, nanograins, nanocavities, liquid and solid skins, interfaces and nanocomposites as a union; (ii) the means of bond energy determination from the measured temperature dependence of band gap and Raman shift; (iii) the factors dominating the super 
elasticity and super plasticity of nanostructures; (iv) the intrinsic and extrinsic competition factors dictating the strongest size of nanostructures and the joint effect of size, composition, temperature, and pressure on the performance of materials.

\subsection{Elasticity and Raman shift}

By definition, ${ }^{44,47}$ it can be derived that the local elastic modulus and the Raman shift from that of a dimer $\omega(1)$ as the reference point at an ith atomic site depend functionally on the bond order $z_{i}$, length $d_{i}$, energy $E_{i}$, and the reduced mass $\mu$ of the dimer atoms $^{43}$ :

$$
\left\{\begin{array}{l}
B_{i}=\left.V \frac{\partial^{2} u(r)}{\partial V^{2}}\right|_{r=d_{i}} \propto \frac{E_{i}}{d_{i}^{3}} \quad \text { (modulus) } \\
\Delta \omega\left(z_{i}\right)=\omega\left(z_{i}\right) \\
\quad-\omega(1) \propto \frac{1}{d_{i}}\left(\frac{E_{i}}{\mu}\right)^{1 / 2} \begin{cases}1 & \text { (Raman Blue shift) } \\
z_{i} & \text { (Raman Red shift) } .\end{cases}
\end{array}\right.
$$

In fact, the elastic modulus is proportional to the binding energy density at equilibrium, according to the dimensionality analysis.

Generally, one can measure the Raman resonance frequency as $\omega_{x}=\omega_{x 0}+\Delta \omega_{x}(\mathrm{x}=D, 2 D, G)$, where $\omega_{x 0}$ is the reference point from which the Raman shift $\Delta \omega_{x}$ proceeds under the applied stimuli. However, the $\omega_{x 0}$ may vary with the frequency of the incident radiation. By expanding the interatomic potential in a Taylor series around its equilibrium and considering the effective atomic $z$, we can derive the vibration frequency shift of the harmonic system,

$$
\begin{aligned}
u(r)= & \sum_{n=0}\left(\frac{d^{n} u(r)}{n ! d r^{n}}\right)_{r=d_{z}}\left(r-d_{z}\right)^{n} \\
\cong & E_{z}+0+\left.\frac{d^{2} u(r)}{2 ! d r^{2}}\right|_{r=d_{z}}\left(r-d_{z}\right)^{2} \\
& +0\left(\left(r-d_{z}\right)^{n \geq 3}\right) \ldots \\
= & E_{z}+\frac{\mu \omega^{2}\left(r-d_{z}\right)^{2}}{2}+0\left(\left(r-d_{z}\right)^{n \geq 3}\right) \cdots
\end{aligned}
$$

From the dimensionality analysis, the term $\left.\frac{\partial u(r)}{\partial r^{2}}\right|_{r=d}$ is proportional to the $\frac{E_{z}}{d^{2}}$. Equaling the vibration energy to the third term in the Taylor series with omitting of the higher-order terms, we have,

$$
\left.\frac{1}{2} \mu(\Delta \omega)^{2} x^{2} \cong \frac{1}{2} \frac{\partial u(r)}{\partial r^{2}}\right|_{r=d} x^{2} \propto \frac{1}{2} \frac{E_{z}}{d^{2}} x^{2}
$$

Observations of the size dependence of the red and blue shift of $\mathrm{TiO}_{2}$ with the size induced abnormal blueshift of the $141 \mathrm{~cm}^{-1}$ vibration indicates that the blueshift arise from dimer vibration while the blue shifted modes involve contributions of all $z$-neighbors of a particular atom.

The elastic modulus and the Raman shift are correlated in dimension as $\left[\Delta \omega\left(z_{i}\right)\right]^{2} /\left[B_{i} d_{i}\right] \equiv 1$ if the $z_{i}$ and $\mu$ are unchanged. These relations apply to any kind of interatomic potential $u(r)$ as the $B_{i}$ and $\Delta \omega\left(z_{i}\right)$ at the $i$ th atomic site are related only to the bond order, length, and energy at the equilibrium.

On the other hand, under the external stimuli of $T$ and $P$ and the variation of $z_{i}$, the length and energy of the specific bond will change, ${ }^{44}$

$$
\left\{\begin{aligned}
d\left(z_{i}, T, P\right)= & \Pi\left(1+\Delta d_{j}\right)=d_{0}\left(1+\left(c_{i}-1\right)\right) \\
& \times \exp \left(\int_{0}^{T} \alpha(t) d t-\int_{0}^{P}\left[\beta-\beta^{\prime} p\right] d p\right) \\
E\left(z_{i}, T, P\right) & =E_{0}\left(1+\sum_{j} \Delta E_{j}\right) \\
& =E_{0}\left[1+\left(c_{i}^{-m}-1\right)+\frac{\Delta E_{P}-\Delta E_{T}}{E_{0}}\right] .
\end{aligned}\right.
$$

$\alpha(t)$ and $\beta(p)$ are, respectively, the efficient of thermal expansion and compressibility. $\beta^{\prime}$ is the nonlinear contribution to the compressibility. $E_{0}$ are the bond energy in the bulk at (atmospheric) $0 \mathrm{~Pa}$ and $0 \mathrm{~K}$. $\Delta E_{T}$ and $\Delta E_{P}$ are the energy perturbation caused by the applied $T$ and $P$. The sum and product proceed over all the $j$ th stimuli. This relation indicates that mechanical compression will shorten and strengthen but thermal vibration will weaken and elongate the representative bond.

(A) Size dependence

Using the core-shell configuration and the LBA approach, ${ }^{25,48}$ we have the following form with $z_{i b}=$ $z_{i} / z_{b}$ for the size dependent elastic modulus, Raman red shift, and Raman blue shift,

$$
\left.\begin{array}{c}
\frac{\Delta B(K)}{B(\infty)} \\
\frac{\Delta[\Delta \omega(K)]}{\Delta \omega(\infty)} \\
\frac{\Delta[\Delta \omega(K)]}{\Delta \omega(\infty)}
\end{array}\right\}=\frac{\Delta_{q}}{K}=\frac{1}{K} \sum_{i \leq 3} \tau c_{i}\left\{\begin{array}{l}
\left(c_{i}^{-(m+3)}-1\right) \\
\left(z_{i b} c_{i}^{-(m / 2+1)}-1\right) \\
\left(c_{i}^{-(\mathrm{m} / 2+1)}-1\right) .
\end{array}\right.
$$


The effective $\mathrm{CN}$ of an atom in the specific $i$ th atomic layer, $z_{i}$, varies with the curvature of a sphere in the form ${ }^{44}: z_{1}=4(1-0.75 / K), z_{2}=z_{1}+2$, and $z_{3}=12$.

(B) Pressure and temperature dependence

For a given specimen of fixed size, we can obtain the analytical form for the $T$ and $P$ dependence of the $B$ and $\Delta \omega$ by combining Eqs. (4) and (5),

$$
\left.\begin{array}{l}
\frac{B(T, P)}{B(0,0)} \\
\frac{\Delta \omega(T, P)}{\Delta \omega(0,0)}
\end{array}\right\}
$$

$$
\cong\left\{\begin{array}{c}
\left(1+\frac{\Delta E_{P}-\Delta E_{T}}{E_{0}}\right) \\
\quad \times \exp \left[3 \left(-\int_{0}^{T} \alpha(t) d t\right.\right. \\
\left.\left.\quad+\int_{0}^{P}\left[\beta-\beta^{\prime} p\right] d p\right)\right] \\
\left(1+\frac{\Delta E_{P}-\Delta E_{T}}{E_{0}}\right)^{1 / 2} \\
\quad \times \exp \left(-\int_{0}^{T} \alpha(t) d t\right. \\
\left.+\int_{0}^{P}\left[\beta-\beta^{\prime} p\right] d p\right) .
\end{array}\right.
$$

The relation $1+x \sim \exp (x)$ is employed when the $x \ll 1$ for the term of bond length. According to Debye approximation, the $T$-induced bond weakening, $\Delta E_{T}$, is the integration of the specific heat $\eta(t)$ from $0 \mathrm{~K}$ to $T$, which follows the relation,

$$
\begin{aligned}
\Delta E_{T} & =\int_{0}^{T} \eta(t) d t=\frac{\int_{0}^{T} C_{v}(t) d t}{z} \\
& =\int_{0}^{T}\left[\int_{0}^{\theta_{D} / T} \frac{9 R}{z}\left(\frac{T}{\theta_{D}}\right)^{3} \frac{y^{4} e^{y}}{\left(e^{y}-1\right)^{2}} d y\right] d t \\
& =\frac{9 R T}{z}\left(\frac{T}{\theta_{D}}\right)^{3} \int_{0}^{\theta_{D} / T} \frac{y^{3} e^{y}}{e^{y}-1} d y,
\end{aligned}
$$

where $R, \theta_{D}$, and $C_{v}$ are the ideal gas constant, the Debye temperature, and the specific heat, respectively. The $y=\theta_{D} / T$ is the reduced form of temperature. $\eta(t)$ is the specific heat per bond, which follows approximately the specific heat of Debye approximation and closes to a constant value of $3 R / z$ ( $R$ is the ideal gas constant) at high temperature.

Likewise, the compressive distortion energy density gain, $\Delta E_{P}$ equals, ${ }^{44}$

$$
\left\{\begin{aligned}
\Delta E_{P} & =-\int_{V_{0}}^{V} p(v) d v=-V_{0} \int_{0}^{P} p(x) \frac{d x}{d p} d p \\
& =V_{0} P^{2}\left[\frac{1}{2} \beta-\frac{2}{3} \beta^{\prime} P\right] \\
x(P) & =V / V_{0} \\
& =1-\beta P+\beta^{\prime} P^{2} ; \quad \frac{d x}{d p}=-\beta+2 \beta^{\prime} P,
\end{aligned}\right.
$$

where $V_{0}$ denotes the volume of any size at zero temperature and zero pressure. The $x(P)$ being a form of the equation of states, matching the measured $x-P$ curve with the $x(P)$ function and the Birch-Mürnaghan (BM) equation, ${ }^{49}$ one can obtain the nonlinear compressibility coefficients, $\beta$ and $\beta^{\prime}$ and the bulk modulus $B_{0}$ and its first-order derivative $B_{0}^{\prime}$ of the specimen. The relation of $\beta B_{0} \cong 1$ holds general for reference. ${ }^{50}$ Substituting Eqs. (8) and (9) into (7), one can have the analytical form for $T$ - and $P$-dependence. In the former, the $E_{c}=z_{b} E_{0}$ and the $\theta_{D}$; in the latter, $E_{d}=E_{0} / V_{0}$ and the $\beta\left(\beta^{\prime}\right)$ are the adjustable parameters. As summarized in Table 1, the analytical forms allow one to extract information such as the bond nature indicator $m$, dimer vibration frequency $\omega(1)$ and its bulk shift $\Delta \omega(\infty)$, atomic cohesive energy $E_{c}=z E_{z}$, Debye temperature $\theta_{D}$, energy density $E_{d}=E_{0} / V_{0}$, and the compressibility, which should be the objectives of the sophisticated measurements.

\section{Experimental and Computational Procedures}

The well-measured and calculated size, ${ }^{2,15}$ temperature, ${ }^{11,12}$ and pressure ${ }^{11}$ dependence of the $B$ and $\Delta \omega$ and the $x-P$ curve ${ }^{13}$ for $\mathrm{TiO}_{2}$ at room temperature allows us to verify the developed solutions. The experimental data were digitized from the following sources. The size dependent elastic modulus was obtained by Dai et al. ${ }^{2}$ in their molecular dynamics simulations. They found that the initial elastic tensile deformation was effected by the reconfiguration of surface atoms and that the 
Table 1. Parameters derived from theoretical reproduction of the size, pressure, and temperature dependence of the bulk modulus and the Raman shift for $\mathrm{TiO}_{2}$.

\begin{tabular}{llcc}
\hline Stimulus & Quantity & Value & References \\
\hline$K$ & Bond nature indicator, $m$ & 5.34 & $5.34^{47}$ \\
& Dimer vibration frequency, $\omega(1)\left(\mathrm{cm}^{-1}\right)$ & $610.25\left(A_{1 \mathrm{~g}} 612\right)$ & - \\
& & $600\left(E_{\mathrm{g}} 639\right)$ & - \\
$T$ & Cohesive energy $E_{c}=z_{b} E_{0}(\mathrm{eV} /$ atom $)$ & $118.35\left(E_{\mathrm{g}} 144\right)$ & $778^{54}$ \\
& Debye temperature,$\theta_{\mathrm{D}}(\mathrm{K})$ & 1.56 & $167 /-^{57}$ \\
$P$ & Bulk modulus, $B_{0} / B_{0}^{\prime}(\mathrm{GPa} /-)$ & 768 & - \\
& Compressibility, $\beta / \beta^{\prime}\left(\mathrm{Gpa}^{-1} / \mathrm{Gpa}^{-2}\right)$ & $6.84 \times 10^{-3}(1.21 \pm 0.05) \times 10^{-4}$ & - \\
\hline
\end{tabular}

nanowires have much better mechanical strength and elasticity than bulk $\mathrm{TiO}_{2}$. The pressure and temperature dependence of the elastic modulus obtained by $\mathrm{Zhu}$ et $a .^{11}$ using the first-principles calculations. They obtained equilibrium structure parameters, bulk modulus $B_{0}$ and its pressure derivative. Swamy ${ }^{15}$ examined the crystallite-sizedependent Raman spectral modifications for rutile $\mathrm{TiO}_{2}$ nanocrystals and compared to the characteristics of the Raman spectra that were reported for nanoscale rutile $\mathrm{TiO}_{2}$ domains/crystallites embedded in thin films. Du et al. ${ }^{12}$ measured the temperature dependence of the Raman modes in anatase $\mathrm{TiO}_{2}$ nanocrystals over the temperature range $77-873 \mathrm{~K}$. With increasing temperature, the frequency of the $E_{\mathrm{g}}$ mode at $639 \mathrm{~cm}^{-1}$ shifts sublinearly to the lower frequencies, however, the frequency of the lowest-frequency $\mathrm{E}$, mode shifts sublinearly to the higher frequencies from $138 \mathrm{~cm}^{-1}$ at $77 \mathrm{~K}$ to $152 \mathrm{~cm}^{-1}$ at $873 \mathrm{~K}$.

In the numerical calculations, we adopt the known effective bond length $d_{0}=0.386 \mathrm{~nm},{ }^{44}$ thermal expansion coefficient $\alpha=8.4 \times 10^{-6} \mathrm{~K}^{-1}, 51$ as input parameters and assume all the data are reliable. Errors in measurements will affect the derived values but not the intrinsic nature and the trends of the observations. Here we are concerned about the nature and trends and aims to verify the proposed approach for extracting meaningful information from the sophisticated measurements. We first calculated the theoretical curve and adjusted the parameters to match the measured and calculated data. The adjustable parameters include the bond nature indicator $m$, dimer vibration frequency $\omega(1)$, cohesive energy $E_{\mathrm{c}}$, Debye temperature $\theta_{\mathrm{D}}$, energy density $E_{\mathrm{d}}$, compressibility and the bulk modulus, and their first-order derivatives $\beta, \beta^{\prime}, B_{0}$, and $B_{0}^{\prime}$ for the output as tabulated in Table 1.

\section{Results and Discussion}

\subsection{Size dependence}

From matching the predicted and the measured/ calculated size dependence of the $Y(K)$, we can determine the $m$ value; from the reproduction of $\Delta \omega(K)$, we can determine the $\omega(1)$ and its bulk shift $\omega(\infty)-\omega(1)$ for the Raman modes showing size-induced red shift. The size-induced blue shift of Raman frequency is governed by a different mechanism that may not involve the coordination number $z$. It has been demonstrated ${ }^{52}$ that the under-coordination-induced bond strain has different effect on the compressive and tensile stress that determines the anisotropic properties and phase transitions in nanocrystalline Barium Titanate.

Figure 1 compares the theoretical reproduction of the measured size dependence of (a) $Y(K),{ }^{2}$ (b)

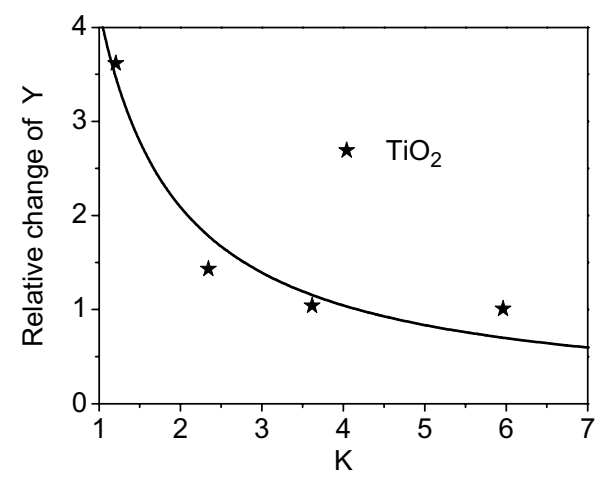

Fig. 1. Theoretical (solid curves) reproduction of the measured size (scattered data $^{2}$ ) dependence of the elastic modulus of nanostructured $\mathrm{TiO}_{2}$. 


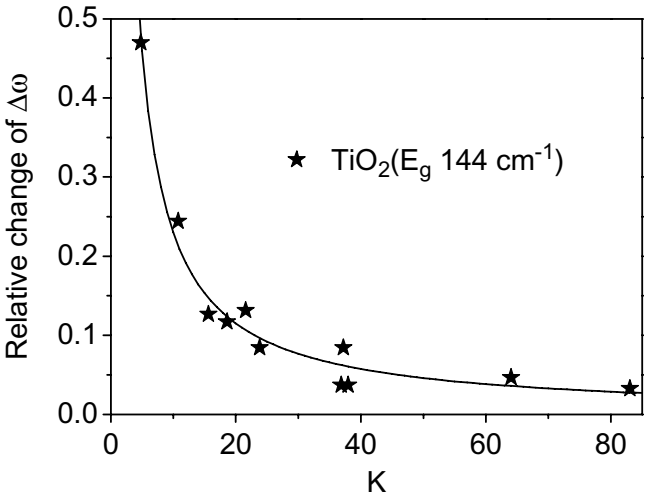

(a)

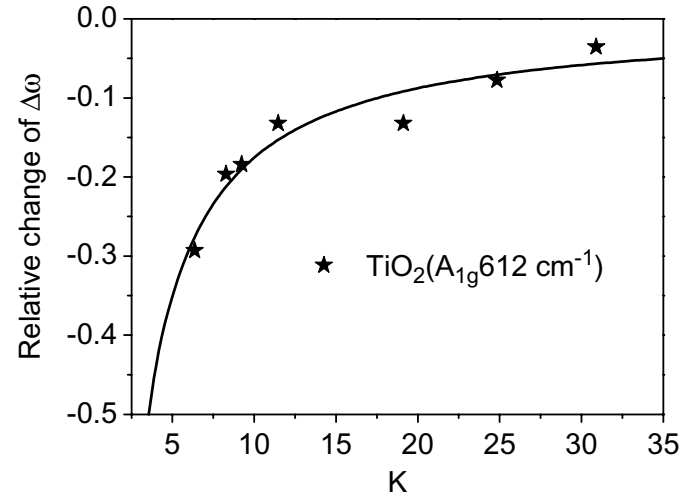

(b)

Fig. 2. Theoretical (solid curves) reproduction of the measured size (scattered data) dependence of the Raman shifts $\Delta \omega(K)^{17}$ of the (a) $E_{\mathrm{g}}\left(144 \mathrm{~cm}^{-1}\right)$ and (b) the $A_{1 \mathrm{~g}}\left(612 \mathrm{~cm}^{-1}\right)$ mode ${ }^{15}$ for $\mathrm{TiO}_{2}$. The paradox in the size-induced shift trends arises from the involvement of the different coordination of the specific atom.

$\Delta \omega(K)^{17}$ of the $E_{\mathrm{g}}\left(144 \mathrm{~cm}^{-1}\right)$ mode of anatase phase, and (c) $\Delta \omega(K)^{15}$ of the $A_{1 \mathrm{~g}}\left(612 \mathrm{~cm}^{-1}\right)$ mode of rutile phase for $\mathrm{TiO}_{2}$ at the room temperature and the atmospheric pressure with the optimized $m$ value of $5.34 .{ }^{47}$ It is observed that the $A_{1 \mathrm{~g}}$ $\left(612 \mathrm{~cm}^{-1}\right)$ mode of rutile phase undergoes a red shift and $E_{\mathrm{g}}\left(144 \mathrm{~cm}^{-1}\right)$ of anatase phase undergoes a blue shift with size reduction. ${ }^{14-16}$ The LFR frequency depends linearly on the inverse of size $K$. When $K$ increases toward infinity, the LFR peaks disappear, which implies that not only the blue shift in the LFR peaks but also the origin for the LFR peaks are purely intergrain interactions. Therefore, the Raman mode red shift arises from atomic cohesive energy weakening of the lower coordinated atoms in the surface region of nanograin, whereas the LFR blue shift is predominated by intergrain interactions. The $E_{\mathrm{g}}\left(144 \mathrm{~cm}^{-1}\right)$ of anatase phase blue shift is dominated by the dimer bond interaction without involvement of the neighboring numbers. Decoding the measured size dependence of the Raman optical shift, we have derived vibrational information on $\mathrm{TiO}_{2}$ dimers $\omega(1)=610.25 \mathrm{~cm}^{-1}$ and their bulk shifts of $1.75 \mathrm{~cm}^{-1}$ for the $A_{1 \mathrm{~g}}$ mode of rutile phase, and $\omega(1)=118.35 \mathrm{~cm}^{-1}$ and their bulk shifts of $25.65 \mathrm{~cm}^{-1}$ for the $E_{\mathrm{g}}$ mode of anatase phase, which is beyond the scope of direct measurement.

\subsection{Temperature dependence}

Matching the temperature dependence of the measurements provides an effective means to determine the Debye temperature and the atomic cohesive energy. ${ }^{53}$ In the $T$-dependent curves, the shoulder is related to the $\theta_{\mathrm{D}}$ and the slope at higher temperature depends on the atomic cohesive energy, $E_{c}=z E_{z}$. Matching the two sets of $B(T)$ and the $\Delta \omega(T)$ data will improve the reliability of the derivatives. The theoretical match of the measured $T$-dependent $B(T)^{11}$ and the $\Delta \omega(T)^{12}$ of the $E_{\mathrm{g}}$ $\left(639 \mathrm{~cm}^{-1}\right)$ mode for anatase phase $\mathrm{TiO}_{2}$ in Fig. 2 leads to the $\omega(1)=600 \mathrm{~cm}^{-1}$ and the $\theta_{\mathrm{D}}$ of $768 \mathrm{~K}$, which is in good agreement with the reported value of $778 \mathrm{~K} .^{54}$ The cohesive energy $E_{\mathrm{c}}$ is derived as $1.56 \mathrm{eV}$. At $T \leq \theta_{\mathrm{D}} / 3$, the relative $B$ and $\Delta \omega$ turns from nonlinear to linear when the temperature is increased. The slow decrease of the $B$ and $\Delta \omega$ at very low temperatures arises from the small $\int_{0}^{T} \eta(t)$ $d t$ values as the specific heat $\eta(t)$ is proportional to $T^{3}$ at very low temperatures. Apparently, this

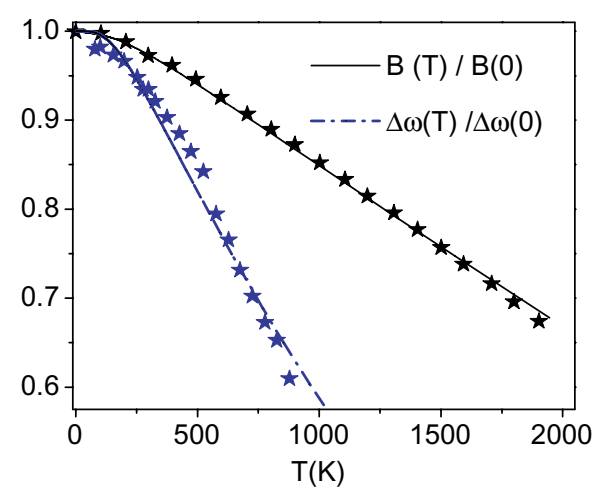

Fig. 3. Theoretical (solid curves) reproduction of the measured $^{11,12} T$ dependence of $B(T)$ and $\Delta \omega(T)$ of $E_{\mathrm{g}}\left(639 \mathrm{~cm}^{-1}\right)$ modes for $\mathrm{TiO}_{2}$. Theoretical matching gives rise to the cohesive energy and Debye temperature as listed in Table 1. 


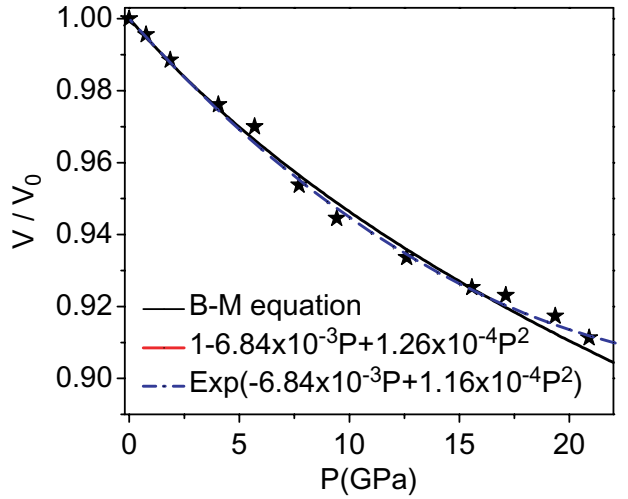

(a)

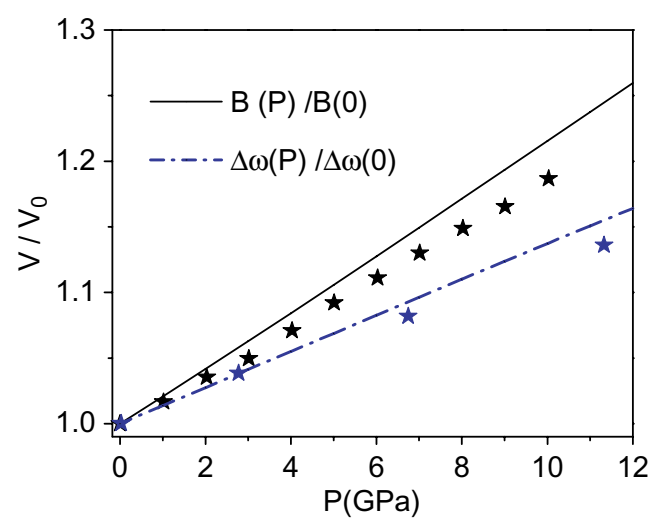

(b)

Fig. 4. (a) Reproduction of the $x-P$ curve $^{13}$ using the polynomial, exponential, and the BM equation gives rise to the compressibility and the bulk modulus, and their first-order derivatives as listed in Table 1. (b) Comparison of the measured and the calculated $P$ dependence of $B(P)^{11}$ and $\Delta \omega(P)^{56}$ of $E_{\mathrm{g}}$ $\left(639 \mathrm{~cm}^{-1}\right)$ modes for $\mathrm{TiO}_{2}$. The calculated curve is based on the nonlinear compressibility from (a).

approach provides information that is beyond the capacity of the model in Eq. (1).

\subsection{Pressure dependence}

Theoretical reproduction of the $P$-dependence will lead to quantitative information of the compressibility and the binding energy density. Figure 4(a) shows that the experimental $x-P$ curve $^{13}$ can be reproduced using the polynomial, the exponential, and the $\mathrm{BM}$ isothermal equation of state, ${ }^{49,55}$ with the optimized $\beta=6.84 \times 10^{-3} \mathrm{GPa}^{-1}, \beta^{\prime}=-1.21 \pm$ $0.05 \times 10^{-4} \mathrm{GPa}^{-2}, B_{0}=143 \mathrm{GPa}$, and $B_{0}^{\prime}=8.86$.

With the gained $\beta$ and $\beta^{\prime}$ values, we calculated the $B(P)$ first. By matching the measurement and the prediction we obtained the binding energy density $0.182 \mathrm{eV} / \AA^{3}$. Then we use the relation $[\Delta \omega]^{2} /[Y d] \equiv 1$ and plot the theoretical $\Delta \omega(P)$ curve as shown in Fig. 4(b). The consistency between theory and prediction of the $P$-dependent $B(P)^{11}$ and $\Delta \omega(P)^{56}$ has been realized for the $E_{\mathrm{g}}$ $\left(639 \mathrm{~cm}^{-1}\right)$ mode for anatase phase $\mathrm{TiO}_{2}$. The slight deviation of the $B(P)$ and $\Delta \omega(P)$ originates from the accuracy in the measurements.

The trends of the $T$ and $P$ dependence of $B$ and $\Delta \omega$ are much the same to that of the $T$ - and $P$-dependent bandgap shift in $\mathrm{ZnO},{ }^{53}$ indicating the interdependence of the Raman shift, bandgap, and elastic modulus, in general.

\section{Conclusion}

We present an analytical expression connecting the macroscopically measurable $B$ and $\Delta \omega$ directly to the bonding identities of the specimen and their response to the intrinsic coordination imperfection and the applied stimuli for deeper insight into the atomistic origin of the size, $T$ - and $P$-induced $B$ and $\Delta \omega$ change in $\mathrm{TiO}_{2}$ through the BOLS correlation and the LBA approach. Exercises lead to derived information of the cohesive energy and Debye temperature, energy density, compressibility and the bulk modulus and their first-order derivatives, and the analytical correlation between the $B$ and $\Delta \omega$. Theoretical reproduction of the measurements reveals that (i) the size effect arises from the under-coordination-induced cohesive energy loss and the energy density gain in the surface up to skin depth; (ii) the thermally softened $B$ and $\Delta \omega$ results from bond expansion and bond weakening due to vibration; and, (iii) the mechanically stiffened $B$ and $\Delta \omega$ results from bond compression and bond strengthening due to mechanical work hardening. The presented approach from the perspective of LBA would provide a useful yet simple way to investigate the mechanical and vibration properties under the stimuli change of atomic coordination and thermal and mechanical activation in other materials as well. The derived quantitative information and the consistent insight and correlation are beyond the scope of currently available approaches. With the developed premise, one can predict the changing trends of all the concerned properties and can derive quantitative information as such from any single measurement alone. 


\section{Acknowledgments}

Financial support from the Special Project for Nanotechnology of Shanghai (No. 1052nm02700) and the Key laboratory of new ceramics and fine processes at Tsinghua University and MOE (RG15/ 09) of Singapore is gratefully acknowledged.

\section{References}

1. G. Guisbiers, O. van Overschelde and M. Wautelet, Appl. Phys. Lett. 92, 103121 (2008).

2. L. Dai, C. H. Sow, C. T. Lim, W. C. D. Cheong and V. B. C. Tan, Nano Lett. 9, 576 (2009).

3. O. Carp, C. L. Huisman and A. Reller, Prog. Solid State Chem. 32, 33 (2004).

4. B. Oregan and M. Gratzel, Nature 353, 737 (1991).

5. U. Bach, D. Lupo, P. Comte, J. E. Moser, F. Weissortel, J. Salbeck, H. Spreitzer and M. Gratzel, Nature 395, 583 (1998).

6. D. J. Dwyer, S. D. Cameron and J. Gland, Surf. Sci. 159, 430 (1985).

7. Y. Matsumoto, M. Murakami, T. Shono, T. Hasegawa, T. Fukumura, M. Kawasaki, P. Ahmet, T. Chikyow, S. Koshihara and H. Koinuma, Science 291, 854 (2001).

8. X. T. Zhang, M. Jin, Z. Y. Liu, D. A. Tryk, S. Nishimoto, T. Murakami and A. Fujishima, J. Phys. Chem. C 111, 14521 (2007).

9. Z. Z. Gu, A. Fujishima and O. Sato, Appl. Phys. Lett. 85, 5067 (2004).

10. Y. J. Wang, C. Jing, L. N. Tan and X. R. Chen, Chin. Phys. Lett. 24, 2642 (2007).

11. J. Zhu, J. X. Yu, Y. J. Wang, X. R. Chen and F. Q. Jing, Chin. Phys. B 17, 2216 (2008).

12. Y. L. Du, Y. Deng and M. S. Zhang, J. Phys. Chem. Solids 67, 2405 (2006).

13. V. Swamy, A. Y. Kuznetsov, L. S. Dubrovinsky, A. Kurnosov and V. B. Prakapenka, Phys. Rev. Lett. 103, 75505 (2009).

14. V. Swamy, A. Kuznetsov, L. S. Dubrovinsky, R. A. Caruso, D. G. Shchukin and B. C. Muddle, Phys. Rev. B 71, 184302 (2005).

15. V. Swamy, Phys. Rev. B 77, 195414 (2008).

16. S. Sahoo, A. K. Arora and V. Sridharan, J. Phys. Chem. C 113, 16927 (2009).

17. V. Swamy, D. Menzies, B. C. Muddle, A. Kuznetsov, L. S. Dubrovinsky, Q. Dai and V. Dmitriev, Appl. Phys. Lett. 88, 243103 (2006).

18. J. G. Guo and Y. P. Zhao, Nanotechnology 18, 295701 (2007).

19. L. X. Zhang and H. C. Huang, Appl. Phys. Lett. 89, 183111 (2006).
20. H. W. Shim, L. G. Zhou, H. C. Huang and T. S. Cale, Appl. Phys. Lett. 86, 151912 (2005).

21. G. Yun and H. S. Park, Phys. Rev. B 79, 195421 (2009).

22. Z. M. Ao, S. Li and Q. Jiang, Appl. Phys. Lett. 93, 081905 (2009).

23. L. G. Zhou and H. C. Huang, Appl. Phys. Lett. 84, 1940 (2009).

24. C. C. Yang and S. Li, Phys. Rev. B 75, 165413 (2007).

25. X. J. Liu, J. W. Li, Z. F. Zhou, L. W. Yang, Z. S. Ma, G. F. Xie, Y. Pan and C. Q. Sun, Appl. Phys. Lett. 94, 131902 (2009).

26. A. Dieguez, A. Romano-Rodriguez, A. Vila and J. R. Morante, J. Appl. Phys. 90, 1550 (2001).

27. E. Anastassakis and E. Liarokapis, J. Appl. Phys. 62, 3346 (1987).

28. K. R. Zhu, M. S. Zhang, Q. Chen and Z. Yin, Phys. Lett. A 340, 220 (2005).

29. K. A. Alim, V. A. Fonoberov, M. Shamsa and A. A. Balandin, J. Appl. Phys. 97, 124313 (2005).

30. J. B. Wachtman, W. E. Tefft, D. G. Lam and C. S. Apstein, Phys. Rev. 122, 1754 (1961).

31. O. L. Anderson, Phys. Rev. 144, 553 (1966).

32. J. Garai and A. Laugier, J. Appl. Phys. 101, 023514 (2007).

33. C. Aku-Leh, J. Zhao, R. Merlin, J. Menendez and M. Cardona, Phys. Rev. B 71, 205211 (2005).

34. J. Serrano, A. H. Romero, F. J. Manjon, R. Lauck, M. Cardona and A. Rubio, Phys. Rev. B 69, 094306 (2004).

35. J. Cui, K. Amtmann, J. Ristein and L. Ley, J. Appl. Phys. 83, 7929 (1998).

36. J. Menndez and M. Cardona, Phys. Rev. B 29, 2051 (1984).

37. R. Cusco, E. Alarcon-Llado, J. Ibanez, L. Artus, J. Jimenez, B. Wang and M. J. Callahan, Phys. Rev. B 75, 165202 (2007).

38. K. Samanta, P. Bhattacharya and R. S. Katiyar, Phys. Rev. B 75, 035208 (2007).

39. J. Liu and Y. K. Vohra, Phys. Rev. Lett. 72, 4105 (1994).

40. J. Zi, H. Büscher, C. Falter, W. Ludwig, K. Zhang and X. Xie, Appl. Phys. Lett. 69, 200 (1996).

41. W. Cheng and S. F. Ren, Phys. Rev. B 65, 205305 (2002).

42. C. Q. Sun, S. Li and B. K. Tay, Appl. Phys. Lett. 82, 3568 (2003).

43. C. Q. Sun, Prog. Solid State Chem. 35, 1 (2007).

44. C. Q. Sun, Prog. Mater. Sci. 54, 179 (2009).

45. W. J. Huang, R. Sun, J. Tao, L. D. Menard, R. G. Nuzzo and J. M. Zuo, Nat. Mater. 7, 308 (2008).

46. W. G. Han and C. T. Zhang, J. Phys.: Condens. Matter 3, 27 (1991). 
47. X. J. Liu, L. W. Yang, Z. F. Zhou, P. K. Chu and C. Q. Sun, J. Appl. Phys. 108, 073503 (2010).

48. J. W. Li, L. W. Yang, Z. F. Zhou, X. J. Liu, G. F. Xie, Y. Pan and C. Q. Sun, J. Phys. Chem. B 114, 1648 (2010).

49. F. Birch, Phys. Rev. 71, 809 (1947).

50. C. Kittel, Introduction to Solid State Physics. (Wiley, New York, 2005).

51. K. Sugiyama and Y. Takeuchi, Z. Kristallogr. 194, 305 (1991).

52. T. Y. Sun, X. H. Wang, H. Wang, X. Q. Zhang, Z. D. Cheng, C. Q. Sun and L. T. Li, J. Am. Ceram. Soc. 93, 2571 (2010).
53. J. W. Li, L. W. Yang, Z. F. Zhou, P. K. Chu, X. H. Wang, J. Zhou, L. T. Li and C. Q. Sun, J. Phys. Chem. C 114, 13370 (2010).

54. A. Y. Wu and R. J. Sladek, Phys. Rev. B 25, 5230 (1982).

55. F. D. Murnaghan, Proc. Nat. Acad. Sci. 30, 244 (1944).

56. V. Swamy, A. Kuznetsov, L. S. Dubrovinsky, R. A. Caruso, D. G. Shchukin and B. C. Muddle, Phys. Rev. B 71, 184302 (2005).

57. B. Chen, H. Z. Zhang, K. A. Dunphy-Guzman, D. Spagnoli, M. B. Kruger, D. V. S. Muthu, M. Kunz, S. Fakra, J. Z. Hu, Q. Z. Guo and J. F. Banfield, Phys. Rev. B 79, 125406 (2009). 